\title{
Structure and stability of recombinant bovine odorant-binding protein: II. Unfolding of the monomeric forms
}

Olga V Stepanenko, Denis O Roginskii, Olesya V Stepanenko, Irina M Kuznetsova, Vladimir N Uversky, Konstantin K Turoverov In a family of monomeric odorant-binding proteins (OBPs), bovine OBP (bOBP), that lacks conserved disulfide bond found in other OBPs, occupies unique niche because of its ability to form domain-swapped dimers. In this study, we analyzed conformational stabilities of the recombinant bOBP and its monomeric variants, the bOBP-Gly $121+$ mutant containing an additional glycine residue after the residue 121 of the bOBP, and the GCC-bOBP mutant obtained from the bOBP-Gly121+ form by introduction of the Trp64Cys/His155Cys double mutation to restore the canonical disulfide bond. We also analyzed the effect of the natural ligand binding on the conformational stabilities of these bOBP variants. Our data are consistent with the conclusion that the unfolding-refolding pathways of the recombinant bOBP and its mutant monomeric forms bOBP-Gly121+ and GCC-bOBP are similar and do not depend on the oligomeric status of the protein. This clearly shows that the information on the unfolding-refolding mechanism is encoded in the structure of the bOBP monomers. However, the process of the bOBP unfolding is significantly complicated by the formation of the domain-swapped dimer, and the rates of the unfolding-refolding reactions essentially depend on the conditions in which the protein is located. 


\section{Structure and Stability of Recombinant Bovine Odorant-Binding}

\section{Protein: II. Unfolding of the Monomeric Forms}

4 Olga V. Stepanenko, ${ }^{1}$ Denis O. Roginskii, ${ }^{1}$ Olesya V. Stepanenko, ${ }^{1}$ Irina M. Kuznetsova, ${ }^{1}$

5 Vladimir N. Uversky,,$^{1,2, *}$ and Konstantin K. Turoverov ${ }^{1,3, *}$

$7{ }^{1}$ Laboratory of structural dynamics, stability and folding of proteins, Institute of Cytology,

8 Russian Academy of Sciences, St. Petersburg, Russia;

$9{ }^{2}$ Department of Molecular Medicine and USF Health Byrd Alzheimer's Research Institute,

10 Morsani College of Medicine, University of South Florida, Tampa, FL, USA;

$11{ }^{3}$ Peter the Great St. Petersburg Polytechnic University, St. Petersburg, Russia

*To whom correspondence should be addressed: VNU, Department of Molecular Medicine,

University of South Florida, 12901 Bruce B. Downs Blvd. MDC07, Tampa, Florida 33612, USA,

Running title: Unfolding of the monomeric bOBP variants 


\section{ABSTRACT}

22 In a family of monomeric odorant-binding proteins (OBPs), bovine OBP (bOBP), that lacks

23 conserved disulfide bond found in other OBPs, occupies unique niche because of its ability to form

24 domain-swapped dimers. In this study, we analyzed conformational stabilities of the recombinant

25 bOBP and its monomeric variants, the bOBP-Gly121+ mutant containing an additional glycine

26 residue after the residue 121 of the bOBP, and the GCC-bOBP mutant obtained from the bOBP-

27 Gly121+ form by introduction of the Trp64Cys/His155Cys double mutation to restore the

28 canonical disulfide bond. We also analyzed the effect of the natural ligand binding on the

29 conformational stabilities of these bOBP variants. Our data are consistent with the conclusion that

30 the unfolding-refolding pathways of the recombinant bOBP and its mutant monomeric forms

31 bOBP-Gly121+ and GCC-bOBP are similar and do not depend on the oligomeric status of the

32 protein. This clearly shows that the information on the unfolding-refolding mechanism is encoded

33 in the structure of the bOBP monomers. However, the process of the bOBP unfolding is

34 significantly complicated by the formation of the domain-swapped dimer, and the rates of the 35 unfolding-refolding reactions essentially depend on the conditions in which the protein is located.

37 Key words: odorant-binding protein; domain swapping; disulfide bond; unfolding-refolding reaction; ligand binding; conformational stability 


\section{INTRODUCTION}

43 Odorant binding proteins (OBPs) are important components of olfactory apparatus in

44 vertebrates where they play a specific role in olfaction by interacting directly with odorants

45 (Xu et al. 2005). OBPs constitute a class of small extracellular proteins in the chemosensory

46 systems of most terrestrial species ranging from drosophila to human. In mammals, OBPs

47 are found at high concentrations $(\sim 10 \mathrm{mM})$ in nasal mucosa of cow (Bignetti et al. 1985;

48 Pelosi et al. 1982), rat (Pevsner et al. 1985), rabbit (Dal Monte et al. 1991), pig (Dal Monte

49 et al. 1991), dog (D'Auria et al. 2006), and humans (Briand et al. 2002). Although they bind

50 different kinds of small and hydrophobic odorant molecules (typically with the affinities in

51 the micromolar range, their inability to discriminate different chemical classes of these

52 molecules suggests that OBPs cannot serve as olfactory receptors (Boudjelal et al. 1996).

53 The precise biological functions of mammalian OBPs are not known as of yet, but it was

54 hypothesized that these proteins can be involved in transport of hydrophobic odorants across

55 the aqueous mucus layer to access the olfactory receptors, or might be involved in the

56 termination of the olfactory signal by removing odorants from the receptors after their

57 stimulation (Bignetti et al. 1987; Pevsner \& Snyder 1990).

58 OBPs constitute a sub-class of lipocalins, which are small extracellular proteins found

59 in gram negative bacteria, plants, invertebrates, and vertebrates. Although lipocalins are

60 known to share limited regions of sequence homology, they do have a common tertiary

61 structure architecture (Flower et al. 2000; Grzyb et al. 2006). The characteristic structural

62 signature of the lipocalin family is a $\beta$-barrel composed by a 9 -stranded anti-parallel $\beta$-sheet

63 with an $\alpha$-helical segment at the C-terminus (Flower et al. 2000). The internal cavity of the

64 lipocalin $\beta$-barrel is the binding site that can interact with the odorant molecules belonging 
65 to different chemical classes (Vincent et al. 2000). Bovine OBP (bOBP) was the first OBP

66 for which crystal structure was solved, and the analysis of this structure revealed that bOBP

67 exists as a domain-swapped dimer (Bianchet et al. 1996; Tegoni et al. 1996). This was in

68 contrast to structures of other lipocalins, including the porcine OBP (pOBP) (Spinelli et al.

69 1998), which are monomeric proteins. The ability of bOBP to form domain-swapped dimers

70 was explained by the absence of a glycine residue at the hinge region linking the $\beta$-barrel to

71 the $\alpha$-helix, and by the lack of the disulfide bridge which is present in all lipocalin sequences

72 identified so far (Ramoni et al. 2002).

This work is dedicated to the analysis of the peculiarities of the GdnHCl-induced

74 unfolding - refolding reactions of the recombinant bOBP and its monomeric mutants, bOBP-

75 Gly121+ and bOBP-Gly121+/W64C/H155C (GCC-bOBP). It continues a series of articles

76 dedicated to the analysis of the effect of the environment (including the presence of crowding

77 agents) on structural properties and conformational stability of bOBP. The mutant protein

78 bOBP-Gly121+ contains an extra glycine residue introduced after the bOBP residue 121 .

79 This substitution was shown to promote monomerization of the bOBP (Stepanenko et al.

$802015)$ likely via increasing the mobility of the loop connecting $\alpha$-helix and $8^{\text {th }} \beta$-strand of the

$81 \beta$-barrel. Substitutions of the residues Trp64 and His156 to cysteines in bOBP-Gly121+

82 generate a mutant form GCC-bOBP, which is expected to have stable monomeric structure

83 due to the restoration of the disulfide bond typically seeing in other OBPs (Ramoni et al.

84 2008). We also investigated the role of the natural ligand in the stabilization of protein

85 structure and looked at how the ligand binding affected the folding-unfolding reaction of 86 these proteins. 


\section{MATHERIAL AND METHODS}

89

Materials

GdnHCl (Nacalai Tesque, Japan), 1-octen-3-ol (OCT; Sigma-Aldrich, USA) and ANS (ammonium salt of 8-anilinonaphtalene-1-sulfonic acid; Fluka, Switzerland) were used without further purification. The protein concentration was $0.1-0.2 \mathrm{mg} / \mathrm{ml}$. The OCT concentration was $10 \mathrm{mM}$. The experiments were performed in $20 \mathrm{mM}$ Na-phosphate-buffered solution at $\mathrm{pH}$ 7.8.

\section{Gene expression and protein purification}

The plasmids pT7-7-bOBP which encodes bOBP and its mutant forms with a poly-histidine tag were used to transform Escherichia coli BL21(DE3) host (Invitrogen) (Stepanenko et al. 2014c). The protein expression was induced by incubating the cells with $0.3 \mathrm{mM}$ of isopropylbeta-D-1-thiogalactopyranoside (IPTG; Fluka, Switzerland) for $24 \mathrm{~h}$ at $37^{\circ} \mathrm{C}$. The recombinant protein was purified with Ni+-agarose packed in HisGraviTrap columns (GE Healthcare, Sweden). The protein purity was determined through SDS-PAGE in $15 \%$ polyacrylamide gel (Laemmli 1970).

\section{Fluorescence spectroscopy}

Fluorescence experiments were performed using a Cary Eclipse spectrofluorometer (Varian, Australia) with microcells FLR (10 x 10 mm; Varian, Australia). Fluorescence lifetime were measured using a "home built" spectrofluorometer with a nanosecond impulse (Stepanenko et al. 2014a; Stepanenko et al. 2012; Turoverov et al. 1998) as well as micro-cells (101.016-QS 5 x 5

$\mathrm{mm}$; Hellma, Germany). Tryptophan fluorescence in the protein was excited at the long-wave 
110 absorption spectrum edge $\left(\lambda_{\mathrm{ex}}=297 \mathrm{~nm}\right)$, wherein the tyrosine residue contribution to the bulk

111 protein fluorescence is negligible (Stepanenko et al. 2015). The fluorescence spectra position and

112 form were characterized using the parameter $A=I_{320} / I_{365}$, wherein $I_{320}$ and $I_{365}$ are the

113 fluorescence intensities at the emission wavelengths 320 and $365 \mathrm{~nm}$, respectively (Turoverov \&

114 Kuznetsova 2003). The values for parameter $A$ and the fluorescence spectrum were corrected for

115 instrument sensitivity. The tryptophan fluorescence anisotropy was calculated using the equation:

$116 r=\left(I_{V}^{V}-G I_{H}^{V}\right) /\left(I_{V}^{V}+2 G I_{H}^{V}\right)$, wherein $I_{V}^{V}$ and $I_{H}^{V}$ are the vertical and horizontal fluorescence

117 intensity components upon excitement by vertically polarized light. $G$ is the relationship between

118 the fluorescence intensity vertical and horizontal components upon excitement by horizontally

119 polarized light $\left(G=I_{V}^{H} / I_{H}^{H}\right), \lambda_{\mathrm{em}}=365 \mathrm{~nm}$ (Turoverov et al. 1998). The fluorescence intensity

120 for the fluorescent dye ANS was recorded at $\lambda_{\mathrm{em}}=480 \mathrm{~nm}\left(\lambda_{\mathrm{ex}}=365 \mathrm{~nm}\right)$.

122 GdnHCl-induced unfolding

123 Protein unfolding was initiated by manually mixing the protein solution $(40 \mu \mathrm{L})$ with a buffer

124 solution $(510 \mu \mathrm{L})$ so that the $\mathrm{GdnHCl}$ concentration was varied from 0.0 to $4.0-5.0 \mathrm{M}$ in the

125 absence or presence of a natural ligand, 1-Octen-3-ol (OCT). The GdnHCl concentration was

126 determined by the refraction coefficient using an Abbe refractometer (LOMO, Russia; (Pace

127 1986)). The dependences of different fluorescent characteristics of the studied proteins on $\mathrm{GdnHCl}$

128 were recorded following protein incubation in a solution with the appropriate denaturant

129 concentration at $4^{\circ} \mathrm{C}$ for different times (see in the text). The protein refolding was initiated by

130 diluting the pre-denatured protein (in $3.0 \mathrm{M} \mathrm{GdnHCl}, 40 \mu \mathrm{L}$ ) with the buffer or denaturant

131 solutions at various concentrations $(510 \mu \mathrm{L})$. The spectrofluorimeter was equipped with a 
132 thermostat that holds the temperature constant at $23^{\circ} \mathrm{C}$.

133

134 Fitting of denaturation curves

135 The equilibrium dependences of the fluorescence intensity at $320 \mathrm{~nm}$ on the $\mathrm{GdnHCl}$

136 concentration were fit using a two-state model:

$$
S=\frac{S_{N}+S_{U} K_{N-U}}{1+K_{N-U}},
$$

138

$$
K_{N-U}=\exp \left(\frac{-\Delta G_{N-U}^{0}+m_{N-U}[D]}{R T}\right)
$$

$$
K_{N-U}=F_{U} / F_{N}=\left(1-F_{N}\right) / F_{N},
$$

140 taking into account

141

$$
S_{N}=a_{N}+b_{N}[D]
$$

$$
S_{U}=a_{U}+b_{U}[D]
$$

143 where $S$ is the fluorescence intensity at the measured GdnHCl concentration; $[D]$ is the guanidine

144 concentration; $m$ is the linear dependence of $\Delta G_{N-U}$ on the denaturant concentration; $\Delta G_{N-U}^{0}$ is the

145 free energy of unfolding at $0 \mathrm{M}$ denaturant; $F_{\mathrm{N}}$ and $F_{\mathrm{U}}$ are the fractions of native and unfolded

146 molecules, respectively; and $S_{N}$ and $S_{U}$ are the signal of the native and unfolded states, respectively;

$147 a_{N}, b_{N}, a_{U}$ and $b_{U}$ are constants needed to fit linear dependences of the $S_{N}$ and $S_{U}$ signals on the

$148 \mathrm{GdnHCl}$ concentration. Fitting was performed using a nonlinear regression with Sigma Plot.

149 To evaluate conformational stability of the studied proteins we took into account that the

150 formation of the native dimeric state of bOBP occurred at moderate $\mathrm{GdnHCl}$ concentration is 
151 followed by full protein unfolding while conformational perturbations of bOBP at low denaturant

152 concentrations were not attributed to the unfolding of the protein globule (see "Results and

153 Discussion" section). As bOBP unfolding is fully reversible the transition from native to unfolded

154 state of the protein was used to calculate $\Delta G_{N-U}$ value. Conformational stability of the bOBP mutant

155 forms was evaluated similarly taking into account that both mutant proteins unfold through the

156 same scheme as bOBP does (see "Results and Discussion" section).

\section{Circular dichroism measurements}

The CD spectra were generated using a Jasco-810 spectropolarimeter (Jasco, Japan). FarUV CD spectra were recorded in a 1-mm path length cell from $260 \mathrm{~nm}$ to $190 \mathrm{~nm}$ with a $0.1 \mathrm{~nm}$

161 step size. For the spectra, we generated 3 scans on average. The CD spectra for the appropriate buffer solution were recorded and subtracted from the protein spectra.

Gel filtration experiments

We performed gel filtration experiments for recombinant bOBPwt and its mutant forms in a buffered solution and with addition of GdnHCl using a Superdex-75 PC 3.2/30 column (GE

167 Healthcare, Sweden) and an AKTApurifier system (GE Healthcare, Sweden). The column was equilibrated with the buffered solution or $\mathrm{GdnHCl}$ at the desired concentration, and $10 \mu \mathrm{l}$ of the

169 protein solution prepared under the same conditions was loaded on the pre-equilibrated column.

170 The change in hydrodynamic dimensions for the studied proteins was evaluated as a change in the

171 protein elution volume. Multiple proteins with known molecular masses (aprotinin $(6.5 \mathrm{kDa})$,

172 ribonuclease $(13.7 \mathrm{kDa})$, carbonic anhydrase $(29 \mathrm{kDa})$, ovalbumin $(43 \mathrm{kDa})$ and conalbumin $(75$ 
$173 \mathrm{kDa}$ ), which are chromatography standards from GE Healthcare) were used to generate the

174 calibration curve.

\section{RESULTS AND DISCUSSION}

178

180

181

182

183

184 naturally monomeric pOBP and the monomeric mutant GCC-bOBP are all characterized by very

190 similar structural flexibility. Since the FlexPred tool provides real-value fluctuations of globular

191 proteins based on their static structures by considering $\mathrm{C} \alpha$ atoms contact number and known B-

192 factor values, the fact that these three proteins, bOBP (PDB ID: 1OBP), GCC-bOBP (PDB ID:

193 2HLV), and pOBP (PDB ID: 1A3Y), are predicted to have similar structural flexibility indicates

194 that mutations introduced to generate the monomeric GCC-bOBP or present in the naturally 
195 monomeric pOBP do not dramatically affect $\mathrm{C} \alpha$ atoms contact number and B-factors of the

196 resulting structures. Note that these observations are in a good agreement with the overall very

197 high structural similarity of these three proteins, the observation supported by the fact that the

198 multiple structural alignment of these three proteins over 136 residues was characterized by the

199 RMSD of $0.89 \AA$ (see Figure 1C). We also compared these results of structure-based flexibility of

200 the bOBP with the propensity of this protein for intrinsic disorder evaluated by PONDR ${ }^{\circledR}$ VSL2B,

201 which is one of the more accurate stand-alone disorder predictors (Fan \& Kurgan 2014; Peng et

202 al. 2005; Peng \& Kurgan 2012). Results of this analysis are also shown in Figure 1D which

203 illustrates that there is generally a very good agreement between the structural flexibility calculated

204 from the protein crystal structure and the propensity of a protein for intrinsic disorder.

Equilibrium unfolding of the recombinant $b O B P$ in the presence of natural ligand

Previously we have shown that the recombinant bOBP, unlike the natural bOBP extracted 208 from the tissues, represents a mixture of monomeric and dimeric forms suggesting that this protein

exists in a stable native-like state with a reduced dimerization capability (Stepanenko et al. 2014c).

210 It has been suggested that the recombinant form of bOBP is characterized by the disturbed package

211 of the $\alpha$-helical region and some $\beta$-strands, which prevents the formation of a native domain-

212 swapped dimer (Stepanenko et al. 2014c). It is likely that the dimer formation via the domain

213 exchange mechanism is a rather complex process that requires specific organization of the

214 secondary and tertiary structure within the monomers. Curiously, recombinant bOBP can form the

215 compact dimeric state under the mild denaturing conditions, namely, in the presence of $1.5 \mathrm{M}$

216 guanidine hydrochloride $(\mathrm{GdnHCl})$ (Stepanenko et al. 2014c). This process requires bOBP

217 restructuring and is accompanied by the formation of a stable, more compact, intermediate state 
218 that is maximally populated at $0.5 \mathrm{M} \mathrm{GdnHCl}$. Noticeably, at $\mathrm{GdnHCl}$ concentrations lower than

$2191.6 \mathrm{M}$ we observed moderate changes in bOBP intrinsic fluorescence and far-UV CD spectra.

220 These changes are not attributed to the bOBP unfolding process but are determined by some local

221 structural changes in the protein globule. The dimeric bOBP in the presence of $1.5 \mathrm{M} \mathrm{GdnHCl}$ is

222 characterized by a highly ordered secondary structure and a highly rigid microenvironment around

223 the tryptophan residues. In the absence of $\mathrm{GdnHCl}$, the recombinant bOBP is in a stable state with

224 features similar to the native dimeric bOBP. Still, recombinant bOBP in the absence of GdnHCl is

225 characterized by a less ordered secondary structure compared to the wild-type bOBP

226 crystallographic data and a more rigid microenvironment of tryptophan residues, which is

227 characterized by a decreased capacity of the recombinant bOBP for dimerization in aqueous

228 solutions. This stable state of the recombinant bOBP state was designated as a "trapped"

229 conformation with incorrect packing of $\alpha$-helices and $\beta$-sheet within the protein globule, which

230 may interfere with the formation of the bOBP native state. The reasons for accumulation of this

231 "trapped" state may lie in a relatively complex domain-swapping dimerization mechanism which

232 is also required for the monomers to be correctly folded. On the other hand, the intermediate state

233 of bOBP structure which is accumulated at $0.5 \mathrm{M} \mathrm{GdnHCl}$ is characterized by the reorganized

234 bOBP structure that has fewer elements of ordered secondary structure, compared with the

235 recombinant bOBP structure both in an aqueous solution and in the solution containing $1.5 \mathrm{M}$

$236 \mathrm{GdnHCl}$. The increase of the $\mathrm{GdnHCl}$ concentration above 1.5 $\mathrm{M}$ induces cooperative unfolding

237 of the recombinant bOBP, which is completed by $\sim 3 \mathrm{M} \mathrm{GdnHCl}$ and is indicated by the

238 simultaneous changes of all structural parameters of bOBP analyzed in this study (Stepanenko et

239 al. 2014c). The half-transition point of this unfolding process at $>2 \mathrm{M} \mathrm{GdnHCl}$ indicates high

240 conformational stability of the recombinant bOBP (Stepanenko et al. 2014c), which is comparable 
241 with the stabilisites of the native (isolated from tissue) bOBP (Mazzini et al. 2002) and pOBP

242 (Staiano et al. 2007; Stepanenko et al. 2008) and is inherent to $\beta$-rich proteins (Stepanenko et al.

243 2012; Stepanenko et al. 2013; Stepanenko et al. 2014b). We have also established that the

244 unfolding of recombinant protein is a completely reversible process, whereas the preceding process

245 of the dimerization of recombinant bOBP is the irreversible event (Stepanenko et al. 2014c).

246 To understand how interaction of the recombinant bOBP with its natural ligand, 1-Octen-3-

$247 \mathrm{ol}(\mathrm{OCT})$ affects this protein, we investigated structural properties and conformational stability of

248 the recombinant bOBP in the presence and absence of OCT. The formation of the protein-ligand

249 complex does not affect the oligomeric status of the protein, since according to the gel filtration

250 analysis the protein in the bOBP/OCT complex continue to exist as a mixture of monomeric and

251 dimeric molecules. However, both monomeric and dimeric forms of the protein become more

252 compact as a result of the OCT binding (Table 1, Figure 2). The GdnHCl-induced unfolding of the

253 bOBP/OCT complex is a rather slow process, since the equilibrium unfolding curves are achieved

254 after incubation of the bOBP/OCT in the presence of different concentrations of the denaturing

255 agent for more than 24 hours. However, the established equilibrium was not affected by further

256 incubation for up to 5 days (Figure 3).

257

Figure 3 shows that the complexity of the bOBP/OCT unfolding is clearly illustrated by the 258 complex shapes of the equilibrium dependencies of various characteristics of this complex on

$259 \mathrm{GdnHCl}$ concentration. This suggests the accumulation of several intermediate states, which are

260 similar to partially folded species found during the equilibrium unfolding of the recombinant bOBP

261 in the absence of OCT. However, compared to the bOBP alone, in the case of the unfolding of

262 bOBP/OCT complex, the accumulation of these intermediate states takes place at higher 263 denaturant concentrations. In fact, a more compact intermediate state of the bOBP/OCT complex 
264 is formed in the concentration range of $0.26-1.0 \mathrm{M} \mathrm{GdnHCl}$, whereas the transition of the

$265 \mathrm{bOBP} / \mathrm{OCT}$ complex to the dimeric state occurs only at 2.0 $\mathrm{M} \mathrm{GdnHCl}$ (Figure 2). Increasing the

$266 \mathrm{GdnHCl}$ concentration over 2.0 M leads to the cooperative unfolding of the bOBP/OCT complex.

267 In comparison with the unfolding of the recombinant bOBP alone, the unfolding of the bOBP/OCT

268 complex occurs in a narrow concentration range, and higher denaturant concentrations are required

269 for complete unfolding of this complex. All these data indicate that formation of the bOBP/OCT

270 complex leads to the substantial stabilization of the protein, but does not affect its unfolding

271 mechanism.

272 To better understand the GdnHCl-induced unfolding of the bOBP/OCT complex, we

273 analyzed the dependence of the 8-anilinonaphthalene-1-sulfonic acid (ANS, also called 1-anilino-

274 8-naphthalenesulfonate), fluorescence on the denaturant concentration. This hydrophobic

275 fluorescent probe is frequently used for the analysis of the presence of solvent-exposed

276 hydrophobic patches in a protein (Stryer 1965) and for the detection of accumulation of partially

277 folded intermediates during equilibrium and kinetic protein unfolding-refolding processes due the

278 ability of ANS to bind to such solvent-exposed hydrophobic patches (which are commonly found

279 in partially folded proteins) and due to the fact that this interaction can be easily detected by the

280 significant increase in the ANS fluorescence intensity and a characteristic blue-shift of its

281 fluorescence maximum (Semisotnov et al. 1991). The shape of the unfolding curve monitored by

282 the GdnHCl-induced changes in the fluorescence intensity of ANS added to the bOBP/OCT was

283 remarkably different from the unfolding curve measured for the recombinant bOBP alone. We

284 observe a smooth continuous decrease in the ANS fluorescence intensity at moderate GdnHCl

285 concentrations, with the ANS fluorescence intensity reaching zero at the denaturant concentrations

286 leading to the formation of the compact dimeric form of bOBP (Figure 3). 

with the ligand binding sites. Therefore, the formation of the bOBP/OCT complex prevents ANS binding. Earlier analysis of the dimeric bOBP structure revealed the presence of an additional ligand binding site at the interface between the monomeric subunits (Bianchet et al. 1996; Ikematsu noticeably weaker than the major ligand binding site located within the $\beta$-barrel (Bianchet et al. 1996; Ikematsu et al. 2005; Pevsner et al. 1985). Our data agree with the presence of an additional 294 ligand binding site in a protein. At the formation of the dimeric bOBP/OCT complex with the native-like compactness at $2.0 \mathrm{M} \mathrm{GdnHCl}$, this additional site is occupied by the ligand, also preventing its interaction with ANS. molecules, which are different from the native dimeric form of the bOBP. Under these conditions, ANS fluorescence intensity in the presence of the bOBP/OCT complex is noticeably lower than the ANS fluorescence recorded for the bOBP alone. These observations suggest that under these conditions the additional ligand binding site of the dimeric bOBP/OCT complex is occupied by ANS, whereas the inner cavity of the barrel is engaged in ligand binding. It is likely that the inability of the natural ligand to interact with the additional weak ligand binding site located between the monomeric subunits can be due to the structural difference of this site in the native dimeric bOBP and in a protein in the original native-like state or an intermediate compact state.

Analysis of the bOBP/OCT refolding from the completely unfolded state revealed that the 
310 fact, during the refolding process, equilibrium values of the analyzed structural characteristics of

311 the bOBP/OCT complex are reached after the incubation of this complex in the presence of the

312 desired $\mathrm{GdnHCl}$ concentration for 72 hrs. No subsequent changes were detected when protein was

313 incubated for 30 days. This analysis revealed the presence of noticeable hysteresis between the

314 curves describing the equilibrium unfolding and refolding of the bOBP/OCT complex in a wide

315 range of the $\mathrm{GdnHCl}$ concentrations. In fact, the equilibrium unfolding and refolding curves

316 coincide only in the vicinity of $2.0 \mathrm{M} \mathrm{GdnHCl}$, where, according to the gel-filtration analysis, the

317 native dimeric state of the bOBP/OCT complex is formed, whereas within the region

318 corresponding to the transition from the native dimeric form to the completely unfolded state of

319 the bOBP/OCT complex, equilibrium curves describing unfolding and refolding of this complex

320 do not coincide.

321 The equilibrium refolding curve describing transition from the unfolded to the compact

322 dimeric state of the bOBP/OCT complex is shifted toward the lower $\mathrm{GdnHCl}$ concentrations in

323 comparison with the equilibrium unfolding curve (Figure 3). However, in comparison with the

324 unfolding of the bOBP alone, this equilibrium refolding curve of the bOBP/OCT complex is still

325 shifted toward higher $\mathrm{GdnHCl}$ concentrations. These data suggest that at the same denaturant

326 concentrations, the fractions of native bOBP formed during the refolding from the completely

327 unfolded state are significantly lower than the fraction of native protein remaining within the

328 region of the bOBP/OCT unfolding. However, once formed, the native protein gains the ability to

329 bind ligand. This hypothesis is supported by the results of the gel-filtration analysis (Figure 2). For

330 example, the elution profiles registered during the unfolding and refolding of the bOBP/OCT

331 complex at $2.5 \mathrm{M} \mathrm{GdnHCl}$ show that under these conditions, more native protein is present during

332 the $\mathrm{bOBP} / \mathrm{OCT}$ unfolding, whereas unfolded species prevail during the refolding of this complex. 
333 Therefore, the effective rates of the formation of various bOBP conformers are significantly

334 different during the unfolding and refolding processes and noticeably depend on the denaturant 335 concentration.

336 It is likely that the same reasons define the irreversibility of the unfolding of the bOBP/OCT 337 complex at low $\mathrm{GdnHCl}$ concentrations. Under these conditions, the rate of the formation of the 338 monomeric bOBP/OCT complex is significantly higher than the rate of the dimeric bOBP/OCT 339 formation. As a result, refolding of the bOBP/OCT complex at the low $\mathrm{GdnHCl}$ concentrations 340 results in the preferential formation of monomeric bOBP/OCT species, whereas under the identical 341 conditions, the unfolding reaction mixture contains roughly equimolar quantities of the 342 bOBP/OCT monomers and dimers (Figures 2 and 3).

344 Equilibrium unfolding of the monomeric bOBP-Gly121+

345 Already at relatively low $\mathrm{GdnHCl}$ concentrations, the monomeric bOBP-Gly121+ is

346 converted to the compact partially folded state with structural characteristics resembling those of 347 the partially folded species accumulated during the equilibrium unfolding of the recombinant

348 bOBP (see Figures 4, 5 and Table 1). This compact intermediate is able to bind ANS and exists in 349 a wide range of the $\mathrm{GdnHCl}$ concentrations (up to about 1.3 M GdnHCl). Subsequent increase in 350 the denaturant concentration promotes transition to a more loose form, which, at the further 351 increase of the $\mathrm{GdnHCl}$ concentration, is converted to the completely unfolded state. This

352 GdnHCl-induced unfolding of the bOBP-Gly121+ is a completely reversible process as evidenced

353 by the coincidence of the equilibrium characteristics of the protein measured at the processes of 354 the bOBP-Gly121+ unfolding and refolding. 
The formation of the bOBP-Gly121+/OCT complex results in a noticeable stabilization of

356

357

358

359

360

361

362

363

364

365

366

367

368

369

370

371

372

373

374

375

376

377

this protein. This is evidenced by the increase in the cooperativity of the unfolding transition, which is also shifted toward higher $\mathrm{GdnHCl}$ concentrations. However, the formation of a complex between the bOBP-Gly121+ and OCT does not affect the unfolding mechanism of this protein.

\section{GdnHCl-induced unfolding of the monomeric GCC-bOBP}

Analysis of the peculiarities of the equilibrium unfolding and refolding processes monitored by the GdnHCl-induced changes in various structural characteristics of the monomeric GCCbOBP suggests that the unfolding of this protein is a completely reversible process accompanied by the formation of partially folded intermediates similar to those observed during the equilibrium unfolding of the recombinant bOBP and its monomeric bOBP-Gly121+ form (see Figures 6, 7 and Table 1). However, although qualitatively unfolded processes of these three proteins are similar, there are some noticeable differences. For example, in comparison with the recombinant bOBP and bOBP-Gly121+ unfolding, a compact intermediate with high ANS affinity is formed at higher denaturant concentrations during the GCC-bOBP unfolding (at 1.0 M GdnHCl). This illustrates higher conformational stability of the disulfide-stabilized GCC-bOBP compared to the recombinant bOBP and its monomeric form bOBP-Gly121+.

GCC-bOBP is further stabilized due to the GCC-bOBP/OCT complex formation. Refolding curves detected by changes in different structural characteristics of this complex and registered after the incubation of the corresponding solutions for one hour coincide with the transition curves describing the equilibrium unfolding of GCC-bOBP, and subsequent incubation of these same solutions for $72 \mathrm{hrs}$ leads to the detectable shift of the transition curves. As a result, equilibrium unfolding and refolding transitions of the GCC-bOBP/OCT complex coincide suggesting that the 
378 unfolding of this protein is a completely reversible process. However, GCC-bOBP becomes able

379 to bind ligand only after the formation of correct native structure stabilized by the disulfide bond.

380 Earlier similar effects were described for other ligand-binding protein, such as the D-glucose/D-

381 galactose-binding protein (GGBP) from E. coli (Stepanenko et al. 2011a; Stepanenko et al. 2009;

382 Stepanenko et al. 2011b). In fact, our analysis of the peculiarities of the GGBP unfolding revealed

383 that ligand binding might constitute a rate-limiting stage of the protein unfolding-refolding

384 process. This phenomenon can be understood considering the fact that the formation of the protein-

385 ligand complex depends on the appearance of the matching configurations between the ligand and

386 the active site of a fully formed native protein (Stepanenko et al. 2011a; Stepanenko et al. 2009;

387 Stepanenko et al. 2011b).

388 Our current analysis revealed that the unfolding of the monomeric complexes bOBP-

389 Gly121+/OCT and GCC-bOBP/OCT is not accompanied by the ANS fluorescence enhancement

390 in the whole range of $\mathrm{GdnHCl}$ concentrations (see Figures 5 and 7). These observations support

391 the hypothesis on the existence of the additional ligand-binding site in the dimeric bOBP.

Table 2 shows that the equilibrium unfolding transition recorded for the recombinant bOBP

393 coincides with that of its monomeric bOBP-Gly121+ form. On the other hand, unfolding transition

394 of the monomeric GCC-bOBP stabilized by the engineered disulfide bond is noticeably shifted to

395 higher denaturant concentrations. Curiously, the unfolding of complexes of all proteins analyzed

396 in this study with natural ligand OCT happens at the same denaturant concentrations.

It is important to note here that the quantitative characterization of the affinity of the bOBP

398 and its mutants to the natural OCT ligand is beyond the scope of this study. In fact, the binding

399 constant of the protein was determined in previous studies (Bianchet et al. 1996; Ikematsu et al.

400 2005; Pevsner et al. 1985). According to published data, binding constant of a natural OCT ligand 
401 to the triple mutant GCC-bOBP is in the micromolar range, which is similar to the affinity of the 402 wild type protein (Ramoni et al. 2008). Amino acid substitutions introduced in mutant proteins, 403 namely the Gly $121+$ insertion and the W64C and H155C replacements, do not affect the ligand404 binding site of the protein. Data reported in the first paper of this series (Stepanenko et al. 2015) 405 revealed that these substitutions do not have significant influence on the protein tertiary and 406 secondary structure. All these data indicate that the mutant forms of the bOBP protein might retain 407 affinity inherent to the wild-type protein.

408 Our data suggest that protein dimerization via the domain-swapping mechanism does not 409 contribute much to the increase in the conformational stability of a protein (at least in the case of 410 the analyzed in this study bOBP), despite the fact that the increased conformational stability was 411 proposed as one of the factors determining the use of this mechanism for dimer and higher 412 oligomer formation (Bennett et al. 1995; Liu \& Eisenberg 2002). In contrast, introduction of a 413 disulfide bond to the structure of a monomeric protein shows significant stabilizing effects. Our 414 data also show that the formation of a protein-ligand complex leads to the significant stabilization 415 of different variants of bOBP and eliminates the original difference in conformational stability 416 caused by their structural differences.

\section{DISCLOSURE}

419 None declared. 


\section{REFERENCES}

422 Bennett MJ, Schlunegger MP, and Eisenberg D. 1995. 3D domain swapping: a mechanism for

423

424

425

426

427

428

429

430

431

432

433

434

435

436

437

438

439

440

441

442

443

444

445

446

447

448

449

450

451

452

453

454

455

456

457

458

459

460

461

462

463

464

465 oligomer assembly. Protein Sci 4:2455-2468.

Bianchet MA, Bains G, Pelosi P, Pevsner J, Snyder SH, Monaco HL, and Amzel LM. 1996. The three-dimensional structure of bovine odorant binding protein and its mechanism of odor recognition. Nat Struct Biol 3:934-939.

Bignetti E, Cavaggioni A, Pelosi P, Persaud KC, Sorbi RT, and Tirindelli R. 1985. Purification and characterisation of an odorant-binding protein from cow nasal tissue. Eur J Biochem 149:227-231.

Bignetti E, Damiani G, De Negri P, Romani R, Avanzini F, Ferrari G, and Rossi GL. 1987. Specificity of an immunoaffinity column for odorant-binding protein from bovine nasal mucosa. Chem Senses 12:601-608.

Boudjelal M, Sivaprasadarao A, and Findlay JB. 1996. Membrane receptor for odour-binding proteins. Biochem J 317 ( Pt 1):23-27.

Briand L, Eloit C, Nespoulous C, Bezirard V, Huet JC, Henry C, Blon F, Trotier D, and Pernollet JC. 2002. Evidence of an odorant-binding protein in the human olfactory mucus: location, structural characterization, and odorant-binding properties. Biochemistry 41:7241-7252.

D'Auria S, Staiano M, Varriale A, Scognamiglio V, Rossi M, Parracino A, Campopiano S, Cennamo N, and Zeni L. 2006. The odorant-binding protein from Canis familiaris: purification, characterization and new perspectives in biohazard assessment. Protein Pept Lett 13:349-352.

Dal Monte M, Andreini I, Revoltella R, and Pelosi P. 1991. Purification and characterization of two odorant-binding proteins from nasal tissue of rabbit and pig. Comp Biochem Physiol $B$ 99:445-451.

Dutta S, Burkhardt K, Young J, Swaminathan GJ, Matsuura T, Henrick K, Nakamura H, and Berman HM. 2009. Data deposition and annotation at the worldwide protein data bank. Mol Biotechnol 42:1-13.

Fan X, and Kurgan L. 2014. Accurate prediction of disorder in protein chains with a comprehensive and empirically designed consensus. J Biomol Struct Dyn 32:448-464.

Flower DR, North AC, and Sansom CE. 2000. The lipocalin protein family: structural and sequence overview. Biochim Biophys Acta 1482:9-24.

Grzyb J, Latowski D, and Strzalka K. 2006. Lipocalins - a family portrait. J Plant Physiol 163:895-915.

Hsin J, Arkhipov A, Yin Y, Stone JE, and Schulten K. 2008. Using VMD: an introductory tutorial. Curr Protoc Bioinformatics Chapter 5:Unit 57.

Ikematsu M, Takaoka D, and Yasuda M. 2005. Odorant binding initially occurring at the central pocket in bovine odorant-binding protein. Biochem Biophys Res Commun 333:12271233.

Jamroz M, Kolinski A, and Kihara D. 2012. Structural features that predict real-value fluctuations of globular proteins. Proteins 80:1425-1435.

Laemmli UK. 1970. Cleavage of structural proteins during the assembly of the head of bacteriophage T4. Nature 227:680-685.

Liu Y, and Eisenberg D. 2002. 3D domain swapping: as domains continue to swap. Protein Sci 11:1285-1299. 
466

467

468

469

470

471

472

473

474

475

476

477

478

479

480

481

482

483

484

485

486

487

488

489

490

491

492

493

494

495

496

497

498

499

500

501

502

503

504

505

506

507

508

509

510

Mazzini A, Maia A, Parisi M, Sorbi RT, Ramoni R, Grolli S, and Favilla R. 2002. Reversible unfolding of bovine odorant binding protein induced by guanidinium hydrochloride at neutral pH. Biochim Biophys Acta 1599:90-101.

Merritt EA, and Bacon DJ. 1977. Raster3D: Photorealistic molecular graphics. . Methods enzymol 277:505-524.

Nolting B. 1999. Protein folding kinetics. In Biophysical Methods, p 111, Springer-Verlag, Berlin-Heidelberg.

Pace CN. 1986. Determination and analysis of urea and guanidine hydrochloride denaturation curves. Methods Enzymol 131:266-280.

Pelosi P, Baldaccini NE, and Pisanelli AM. 1982. Identification of a specific olfactory receptor for 2-isobutyl-3-methoxypyrazine. Biochem J 201:245-248.

Peng K, Vucetic S, Radivojac P, Brown CJ, Dunker AK, and Obradovic Z. 2005. Optimizing long intrinsic disorder predictors with protein evolutionary information. J Bioinform Comput Biol 3:35-60.

Peng ZL, and Kurgan L. 2012. Comprehensive comparative assessment of in-silico predictors of disordered regions. Curr Protein Pept Sci 13:6-18.

Pevsner J, and Snyder SH. 1990. Odorant-binding protein: odorant transport function in the vertebrate nasal epithelium. Chem Senses 15:217-222.

Pevsner J, Trifiletti RR, Strittmatter SM, and Snyder SH. 1985. Isolation and characterization of an olfactory receptor protein for odorant pyrazines. Proc Natl Acad Sci U S A 82:30503054.

Ramoni R, Spinelli S, Grolli S, Conti V, Merli E, Cambillau C, and Tegoni M. 2008. Deswapping bovine odorant binding protein. Biochim Biophys Acta 1784:651-657.

Ramoni R, Vincent F, Ashcroft AE, Accornero P, Grolli S, Valencia C, Tegoni M, and Cambillau C. 2002. Control of domain swapping in bovine odorant-binding protein. Biochem J 365:739-748.

Semisotnov GV, Rodionova NA, Razgulyaev OI, Uversky VN, Gripas AF, and Gilmanshin RI. 1991. Study of the "molten globule" intermediate state in protein folding by a hydrophobic fluorescent probe. Biopolymers 31:119-128.

Shatsky M, Nussinov R, and Wolfson HJ. 2004. A method for simultaneous alignment of multiple protein structures. Proteins 56:143-156.

Spinelli S, Ramoni R, Grolli S, Bonicel J, Cambillau C, and Tegoni M. 1998. The structure of the monomeric porcine odorant binding protein sheds light on the domain swapping mechanism. Biochemistry 37:7913-7918.

Staiano M, D'Auria S, Varriale A, Rossi M, Marabotti A, Fini C, Stepanenko OV, Kuznetsova IM, and Turoverov KK. 2007. Stability and dynamics of the porcine odorant-binding protein. Biochemistry 46:11120-11127.

Stepanenko OV, Bublikov GS, Stepanenko OV, Shcherbakova DM, Verkhusha VV, Turoverov KK, and Kuznetsova IM. 2014a. A knot in the protein structure - probing the nearinfrared fluorescent protein iRFP designed from a bacterial phytochrome. Febs $J$ 281:2284-2298.

Stepanenko OV, Fonin AV, Stepanenko OV, Morozova KS, Verkhusha VV, Kuznetsova IM, Turoverov KK, Staiano M, and D'Auria S. 2011a. New insight in protein-ligand interactions. 2. Stability and properties of two mutant forms of the D-galactose/Dglucose-binding protein from E. coli. J Phys Chem B 115:9022-9032. 
511 Stepanenko OV, Fonin AV, Stepanenko OV, Staiano M, D'Auria S, Kuznetsova IM, and

512

513

514

515

516

517

518

519

520

521

522

523

524

525

526

527

528

529

530

531

532

533

534

535

536

537

538

539

540

541

542

543

544

545

546

547

548

549

550

551

552

553

554

555

Turoverov KK. 2015. Tryptophan residue of the D-galactose/D-glucose-binding protein from E. Coli localized in its active center does not contribute to the change in intrinsic fluorescence upon glucose binding. J Fluoresc 25:87-94.

Stepanenko OV, Marabotti A, Kuznetsova IM, Turoverov KK, Fini C, Varriale A, Staiano M, Rossi M, and D'Auria S. 2008. Hydrophobic interactions and ionic networks play an important role in thermal stability and denaturation mechanism of the porcine odorantbinding protein. Proteins 71:35-44.

Stepanenko OV, Povarova OI, Stepanenko OV, Fonin AV, Kuznetsova IM, Turoverov KK, Staiano M, and D'Auria S. 2009. Structure and stability of D-galactose/D-glucosebinding protein. The role of D-glucose binding and Ca ion depletion. Spectrosc Biomed Appl 24 355-359.

Stepanenko OV, Stepanenko OV, Kuznetsova IM, Shcherbakova DM, Verkhusha VV, and Turoverov KK. 2012. Distinct effects of guanidine thiocyanate on the structure of superfolder GFP. PLoS One 7:e48809.

Stepanenko OV, Stepanenko OV, Kuznetsova IM, Verkhusha VV, and Turoverov KK. 2013. Beta-barrel scaffold of fluorescent proteins: folding, stability and role in chromophore formation. Int Rev Cell Mol Biol 302:221-278.

Stepanenko OV, Stepanenko OV, Kuznetsova IM, Verkhusha VV, and Turoverov KK. 2014 b. Sensitivity of superfolder GFP to ionic agents. PLoS One 9:e110750.

Stepanenko OV, Stepanenko OV, Povarova OI, Fonin AV, Kuznetsova IM, Turoverov KK, Staiano M, Varriale A, and D'Auria S. 2011b. New insight into protein-ligand interactions. The case of the D-galactose/D-glucose-binding protein from Escherichia coli. J Phys Chem B 115:2765-2773.

Stepanenko OV, Stepanenko OV, Staiano M, Kuznetsova IM, Turoverov KK, and D'Auria S. 2014c. The quaternary structure of the recombinant bovine odorant-binding protein is modulated by chemical denaturants. PLoS One 9:e85169.

Stepanenko OV, Roginskii DO, Stepanenko OV, Kuznetsova IM, Uversky VN, and Turoverov KK. 2015. Structure and stability of recombinant bovine odorant-binding protein: I. Design and analysis of monomeric mutants. PeerJ. Submitted.

Stryer L. 1965. The interaction of a naphthalene dye with apomyoglobin and apohemoglobin. A fluorescent probe of non-polar binding sites. J Mol Biol 13:482-495.

Tegoni M, Ramoni R, Bignetti E, Spinelli S, and Cambillau C. 1996. Domain swapping creates a third putative combining site in bovine odorant binding protein dimer. Nat Struct Biol 3:863-867.

Turoverov KK, Biktashev AG, Dorofeiuk AV, and Kuznetsova IM. 1998. [A complex of apparatus and programs for the measurement of spectral, polarization and kinetic characteristics of fluorescence in solution]. Tsitologiia 40:806-817.

Turoverov KK, and Kuznetsova IM. 2003. Intrinsic fluorescence of actin. J Fluorescence 13:4157.

Vincent F, Spinelli S, Ramoni R, Grolli S, Pelosi P, Cambillau C, and Tegoni M. 2000. Complexes of porcine odorant binding protein with odorant molecules belonging to different chemical classes. J Mol Biol 300:127-139.

$\mathrm{Xu}$ P, Atkinson R, Jones DN, and Smith DP. 2005. Drosophila OBP LUSH is required for activity of pheromone-sensitive neurons. Neuron 45:193-200.

PeerJ reviewing PDF | (2015:10:7277:2:0:NEW 16 Dec 2015) 


\section{FIGURE LEGENDS}

558 Figure 1. Analysis of the 3D structure of bOBP. Crystal 3D structures of natural bOBP $(\boldsymbol{A})$ and

559

560

561

562

563

564

565

566

567

568

569

570

571

572

573

574

575

576

577

578

579

580

581

582

583

584

585

586

587

588

589

590

591

592

593

594

595

596

597

598

599

600

601 monomeric mutant form GCC-bOBP $(\boldsymbol{B})$. The individual subunits in the bOBP are in gray and orange. In the GCC-bOBP short $\alpha$-helical segment that followed by the 9th $\beta$-strand and the disordered C-terminal region of the protein are drown in orange. The tryptophan residues are indicated in red and blue in the different subunits of bOBP and in blue in GCC-bOBP. The Gly $121+$ residue which donates the increased mobility of the loop connecting $\alpha$-helix and 8 th $\beta$-strand of the $\beta$-barrel and promotes the formation of a monomeric fold of the mutant protein bOBPGly121+ is in green. Two cysteine residues Cys 64 and Cys 156 in GCC-bOBP, which are believed to stabilize monomeric structure due to the disulfide bond formation are shown in yellow. The drawing of bOBP and GCC-bOBP was generated based on the 1OBP (Tegoni et al. 1996) and 2HLV files (Ramoni et al. 2008) from PDB (Dutta et al. 2009) using the graphic software VMD (Hsin et al. 2008) and Raster3D (Merritt \& Bacon 1977). Plot $\boldsymbol{C}$ represents the results of the multiple structural alignment of bOBP (PDB ID: 1OBP, blue structure), GCC-bOBP (PDB ID: $2 \mathrm{HLV}$, red structure), and naturally monomeric pOBP (PDB ID: 1A3Y, green structure) using the MultiProt algorithm (http://bioinfo3d.cs.tau.ac.il/MultiProt/) (Shatsky et al. 2004). The drawing was generated using the graphic software VMD (Hsin et al. 2008). Plot $\boldsymbol{D}$ compares flexibility profiles obtained from crystal structures of bOBP (PDB ID: 1OBP, black and red lines for the chains $\mathrm{A}$ and $\mathrm{B}$ ), naturally monomeric pOBP (PDB ID: 1A3Y, green and yellow lines for the chains A and B) and monomeric mutant GCC-bOBP (PDB ID: 2HLV, blue line) with the intrinsic disorder propensity of the bOBP (UniProt ID: P07435, pink dashed line). Flexibility profiles were obtained using the FlexPred software available at http://kiharalab.org/flexPred/ (Jamroz et al. 2012), whereas intrinsic disorder propensity of the bOBP (UniProt ID: P07435) was evaluated using the PONDR ${ }^{\circledR}$ VSL2 algorithm (Peng et al. 2005).

Figure 2. The changes of hydrodynamic dimensions of recombinant bOBP $(A$ and $B)$ and its complex with ligand bOBP/OCT ( $C$ and $D$ ) in different structural states. The elution profiles for bOBP and bOBP/OCT were recorded during the protein denaturation $(\boldsymbol{A}$ and $\boldsymbol{C})$ and renaturation from unfolded states $(\boldsymbol{B}$ and $\boldsymbol{D})$ induced by $\mathrm{GdnHCl}$. The elution profiles for bOBP were measured after pre-incubation of the protein and the solution of $\mathrm{GdnHCl}$ in desired concentration for $24 \mathrm{~h}(\boldsymbol{A}$ and $\boldsymbol{B})$, while in the case of bOBP/OCT the incubation time was extended to $72-84 \mathrm{~h}$ for denaturation $(\boldsymbol{C})$ and 6 days for renaturation $(\boldsymbol{D})$. The figures on the curves are the $\mathrm{GdnHCl}$ concentrations.

Figure 3. bOBP and bOBP/OCT conformational changes induced by GdnHCl. $A$ : changes in fluorescence intensity at $320 \mathrm{~nm}, \lambda_{\mathrm{ex}}=297 \mathrm{~nm} ; \boldsymbol{B}$ : changes in parameter $A, \lambda_{\mathrm{ex}}=297 \mathrm{~nm}$; $\boldsymbol{C}$ : changes in fluorescence anisotropy at the emission wavelength $365 \mathrm{~nm}, \lambda_{\mathrm{ex}}=297 \mathrm{~nm} ; \boldsymbol{D}$ : changes in the ellipticity at $222 \mathrm{~nm} ; \boldsymbol{E}$ : changes in the ANS fluorescence intensity at $\lambda_{\mathrm{ex}}=365 \mathrm{~nm}, \boldsymbol{\lambda}_{\mathrm{em}}=480 \mathrm{~nm}$. The statistical errors for fluorescence measurements were assessed and were shown to fall within the range of $0.2-1 \%$.

The measurements were preceded by incubating the protein in a solution with the appropriate $\mathrm{GdnHCl}$ concentration at $4^{\circ} \mathrm{C}$ for 24 (red circles) in the case of bOBP. The open symbols indicate unfolding, whereas the closed symbols represent refolding. While studying the folding of bOBP/OCT (squares), the solution of complex of the protein with its ligand were incubated in a solution with the appropriate $\mathrm{GdnHCl}$ concentration at $4^{\circ} \mathrm{C}$ for less than $24 \mathrm{~h}$ (open brown squares), 
602 up to $120 \mathrm{~h}$ (open pink squares) at the protein denaturation, and $1 \mathrm{~h}$ (closed brown squares) and 72 $603 \mathrm{~h}-30$ days (closed pink squares) at the protein renaturation.

604

605

Figure 4. The changes of hydrodynamic dimensions of recombinant bOBP-Gly121+ $(A$ and

606 $B)$ and its complex with ligand bOBP-Gly121+/OCT $(C$ and $D)$ in different structural states.

607 The elution profiles for bOBP-Gly121+ and bOBP-Gly121+/OCT were recorded during the

608

609 protein denaturation $(\boldsymbol{A}$ and $\boldsymbol{C}$ ) and renaturation from unfolded states ( $\boldsymbol{B}$ and $\boldsymbol{D})$ induced by $\mathrm{GdnHCl}$. The elution profiles for bOBP-Gly121+ were measured after pre-incubation of the

610 protein and the solution of $\mathrm{GdnHCl}$ in desired concentration for $24 \mathrm{~h}(\boldsymbol{A}$ and $\boldsymbol{B})$, while in the case of bOBP-Gly121+/OCT the incubation time was extended to $72-84 \mathrm{~h}$ for denaturation $(\boldsymbol{C})$ and 6

612

613

614

615 days for renaturation $(\boldsymbol{D})$. The figures on the curves are the $\mathrm{GdnHCl}$ concentrations.

Figure 5. bOBP-Gly121+ and bOBP-Gly121+/OCT conformational changes induced by GdnHCl. $\boldsymbol{A}$ : changes in fluorescence intensity at $320 \mathrm{~nm}, \lambda_{\mathrm{ex}}=297 \mathrm{~nm} ; \boldsymbol{B}$ : changes in parameter $A, \lambda_{\mathrm{ex}}=297 \mathrm{~nm} ; \boldsymbol{C}$ : changes in fluorescence anisotropy at the emission wavelength $365 \mathrm{~nm}, \lambda_{\mathrm{ex}}=297$ $\mathrm{nm} ; \boldsymbol{D}$ : changes in the ellipticity at $222 \mathrm{~nm} ; \boldsymbol{E}$ : changes in the ANS fluorescence intensity at $\lambda_{\mathrm{ex}}=365 \mathrm{~nm}, \lambda_{\mathrm{em}}=480 \mathrm{~nm}$. The statistical errors for fluorescence measurements were assessed and were shown to fall within the range of $0.2-1 \%$.

620 The measurements were preceded by incubating the protein in a solution with the appropriate $\mathrm{GdnHCl}$ concentration at $4^{\circ} \mathrm{C}$ for 24 (dark green circles) in the case of bOBP-Gly $121+$. The open symbols indicate unfolding, whereas the closed symbols represent refolding. While studying the folding of bOBP-Gly121+/OCT (squares), the solution of complex of the protein with its ligand were incubated in a solution with the appropriate $\mathrm{GdnHCl}$ concentration at $4^{\circ} \mathrm{C}$ for less than $24 \mathrm{~h}$

625

626

627

628 (open dark yellow squares), up to $72 \mathrm{~h}$ (open light green squares) at the protein denaturation, and $1 \mathrm{~h}$ (closed dark yellow squares) and $72 \mathrm{~h}$ (closed light green squares) at the protein renaturation.

629

Figure 6. The changes of hydrodynamic dimensions of recombinant GCC-bOBP ( $A$ and $B$ ) and its complex with ligand GCC-bOBP/OCT $(C$ and $D)$ in different structural states. The elution profiles for GCC-bOBP and GCC-bOBP/OCT were recorded during the protein denaturation ( $\boldsymbol{A}$ and $\boldsymbol{C}$ ) and renaturation from unfolded states $(\boldsymbol{B}$ and $\boldsymbol{D})$ induced by $\mathrm{GdnHCl}$. The elution profiles for GCC-bOBP were measured after pre-incubation of the protein and the solution of $\mathrm{GdnHCl}$ in desired concentration for $24 \mathrm{~h}(\boldsymbol{A}$ and $\boldsymbol{B})$, while in the case of GCC-bOBP/OCT the incubation time was extended to $72-84 \mathrm{~h}$ for denaturation $(\boldsymbol{C})$ and 6 days for renaturation $(\boldsymbol{D})$.

636

637 The figures on the curves are the $\mathrm{GdnHCl}$ concentrations. $\boldsymbol{A}$ : changes in fluorescence intensity at $320 \mathrm{~nm}, \lambda_{\mathrm{ex}}=297 \mathrm{~nm} ; \boldsymbol{B}$ : changes in parameter $A, \lambda_{\text {ex }}=297$ $\mathrm{nm} ; \boldsymbol{C}$ : changes in fluorescence anisotropy at the emission wavelength $365 \mathrm{~nm}, \lambda_{\text {ex }}=297 \mathrm{~nm} ; \boldsymbol{D}$ : changes in the ellipticity at $222 \mathrm{~nm} ; \boldsymbol{E}$ : changes in the ANS fluorescence intensity at $\lambda_{\mathrm{ex}}=365 \mathrm{~nm}$, $\lambda_{\mathrm{em}}=480 \mathrm{~nm}$. The statistical errors for fluorescence measurements were assessed and were shown

642 to fall within the range of $0.2-1 \%$.

643 The measurements were preceded by incubating the protein in a solution with the appropriate

644

645 $\mathrm{GdnHCl}$ concentration at $4^{\circ} \mathrm{C}$ for 24 (blue circles) in the case of GCC-bOBP. The open symbols indicate unfolding, whereas the closed symbols represent refolding. While studying the folding of GCC-bOBP/OCT (squares), the solution of complex of the protein with its ligand were incubated in a solution with the appropriate $\mathrm{GdnHCl}$ concentration at $4^{\circ} \mathrm{C}$ for less than $24 \mathrm{~h}$ (open dark blue 
648 squares), up to $72 \mathrm{~h}$ (open light blue squares) at the protein denaturation, and $1 \mathrm{~h}$ (closed dark blue 649 squares) and $72 \mathrm{~h}$ (closed light blue squares) at the protein renaturation. 


\section{Table $\mathbf{1}$ (on next page)}

Table 1

Hydrodynamic dimensions of recombinant bOBPwt and its mutant forms in the absence and in the presence of natural ligand OCT in different structural states. 
2 Table 1. Hydrodynamic dimensions of recombinant bOBPwt and its mutant forms in the absence 3 and in the presence of natural ligand OCT in different structural states.

4

\begin{tabular}{|l|c|c|c|}
\hline & GdnHCl, M & First peak, $\mathrm{kDa}$ & Second peak, kDa \\
\hline bOBPwt & 0.0 & 43.9 & 23.8 \\
& 0.5 & 34.0 & 19.3 \\
& 1.5 & 43.6 & 21.5 \\
bOBPwt/OCT & 0.0 & 39.6 & 17.0 \\
& 0.55 & 27.2 & \\
\hline bOBP/Gly121+ & 1.7 & 39.6 & \\
& 0.0 & 23.6 & \\
\hline bOBP/Gly121+/OCT & 0.25 & 17.8 & \\
& $1.5-1.9$ & $24.8-28.5$ & \\
& 0.0 & 21.5 & \\
\hline GCC-bOBP & $0.24-0.5$ & $15.5-16.2$ & \\
& $1.7-2.0$ & $24.7-28.5$ & \\
& 0.0 & 23.6 & \\
\hline GCC-bOBP/OCT & 0.25 & 17.8 & \\
& $1.1-1.82$ & $22.5-25.9$ & \\
& 0.0 & 22.5 & \\
& 0.27 & 16.9 & \\
& $1.5-2.0$ & $22.5-27.2$ & \\
\hline
\end{tabular}




\section{Table 2 (on next page)}

Table 2

Thermodynamic parameters of $\mathrm{GdnHCl}$-induced denaturation of bOBP, its mutant variants and their complexes with octen-3-ol (OCT) 
2 Table 2. Thermodynamic parameters of GdnHCl-induced denaturation of bOBP, its mutant 3 variants and their complexes with octen-3-ol (OCT)

4

\begin{tabular}{|c|c|c|c|}
\hline protein & $\begin{array}{c}m \\
\left(\mathrm{~kJ} \mathrm{~mol}^{-1} \mathrm{M}^{-1}\right)\end{array}$ & $C_{m}(\mathrm{M})^{\mathrm{a}}$ & $\begin{array}{c}\Delta \boldsymbol{G}^{\mathbf{0}}{ }_{\mathbf{N}-\mathbf{U}^{-1}}^{\mathrm{b}} \\
\left(\mathrm{kJ} \mathrm{mol}^{-1}\right)\end{array}$ \\
\hline bOBP & $3.7 \pm 0.2$ & $2.1 \pm 0.1$ & $7.7 \pm 0.6$ \\
\hline bOBP/OCT & $4.1 \pm 0.2$ & $2.7 \pm 0.1$ & $11.0 \pm 0.5$ \\
\hline bOBP/Gly121+ & $2.8 \pm 0.2$ & $2.0 \pm 0.1$ & $5.5 \pm 0.4$ \\
\hline bOBP/Gly121+/OCT & $5.3 \pm 0.2$ & $2.6 \pm 0.1$ & $14.0 \pm 0.5$ \\
\hline GCC-bOBP & $2.5 \pm 0.3$ & $2.3 \pm 0.1$ & $5.8 \pm 0.7$ \\
\hline GCC-bOBP/OCT & $4.5 \pm 0.3$ & $2.7 \pm 0.1$ & $12.0 \pm 0.9$ \\
\hline
\end{tabular}

5

$6 \quad{ }^{a} C_{m}$ is the denaturant concentration at midpoint of conformational transition

$7 \quad \mathrm{~b}$ The fluorescence signals of the folded and unfolded states were approximated by linear

8 dependences as function of denaturant concentration (Nolting, 1999).

9

10

11 


\section{1}

Analysis of the 3D structure of bOBP.

Crystal 3D structures of natural bOBP $(\boldsymbol{A})$ and monomeric mutant form GCC-bOBP $(\boldsymbol{B})$. The individual subunits in the bOBP are in gray and orange. In the GCC-bOBP short $\alpha$-helical segment that followed by the 9th $\beta$-strand and the disordered C-terminal region of the protein are drown in orange. The tryptophan residues are indicated in red and blue in the different subunits of bOBP and in blue in GCC-bOBP. The Gly 121+ residue which donates the increased mobility of the loop connecting $\alpha$-helix and 8 th $\beta$-strand of the $\beta$-barrel and promotes the formation of a monomeric fold of the mutant protein bOBP-Gly121+ is in green. Two cysteines residues Cys 64 and Cys 156 in GCC-bOBP, which are believed to stabilize monomeric structure due to the disulfide bond formation are in yellow. The drawing of bOBP and GCC-bOBP was generated based on the 1OBP (Tegoni et al. 1996) and 2HLV files (Ramoni et al. 2008) from PDB (Dutta et al. 2009) using the graphic software VMD (Hsin et al. 2008) and Raster3D (Merritt \& Bacon 1977). Plot $\boldsymbol{C}$ represents the results of the multiple structural alignment of bOBP (PDB ID: 1OBP, blue structure), GCC-bOBP (PDB ID: 2HLV, red structure), and naturally monomeric pOBP (PDB ID: 1A3Y, green structure) using the MultiProt algorithm ( http://bioinfo3d.cs.tau.ac.il/MultiProt/ ) (Shatsky et al. 2004). The drawing was generated using the graphic software VMD (Hsin et al. 2008). Plot $\boldsymbol{D}$ compares flexibility profiles obtained from crystal structures of bOBP (PDB ID: 1OBP, black and red lines for the chains $A$ and $B$ ), naturally monomeric pOBP (PDB ID: 1A3Y, green and yellow lines for the chains A and B) and monomeric mutant GCC-bOBP (PDB ID: 2HLV, blue line) with the intrinsic disorder propensity of the bOBP (UniProt ID: P07435, pink dashed line). Flexibility profiles were obtained using the FlexPred software available at http://kiharalab.org/flexPred/ (Jamroz et al. 2012), whereas intrinsic disorder propensity was evaluated using the PONDR $®$ VSL2 algorithm (Peng et al. 2005). 

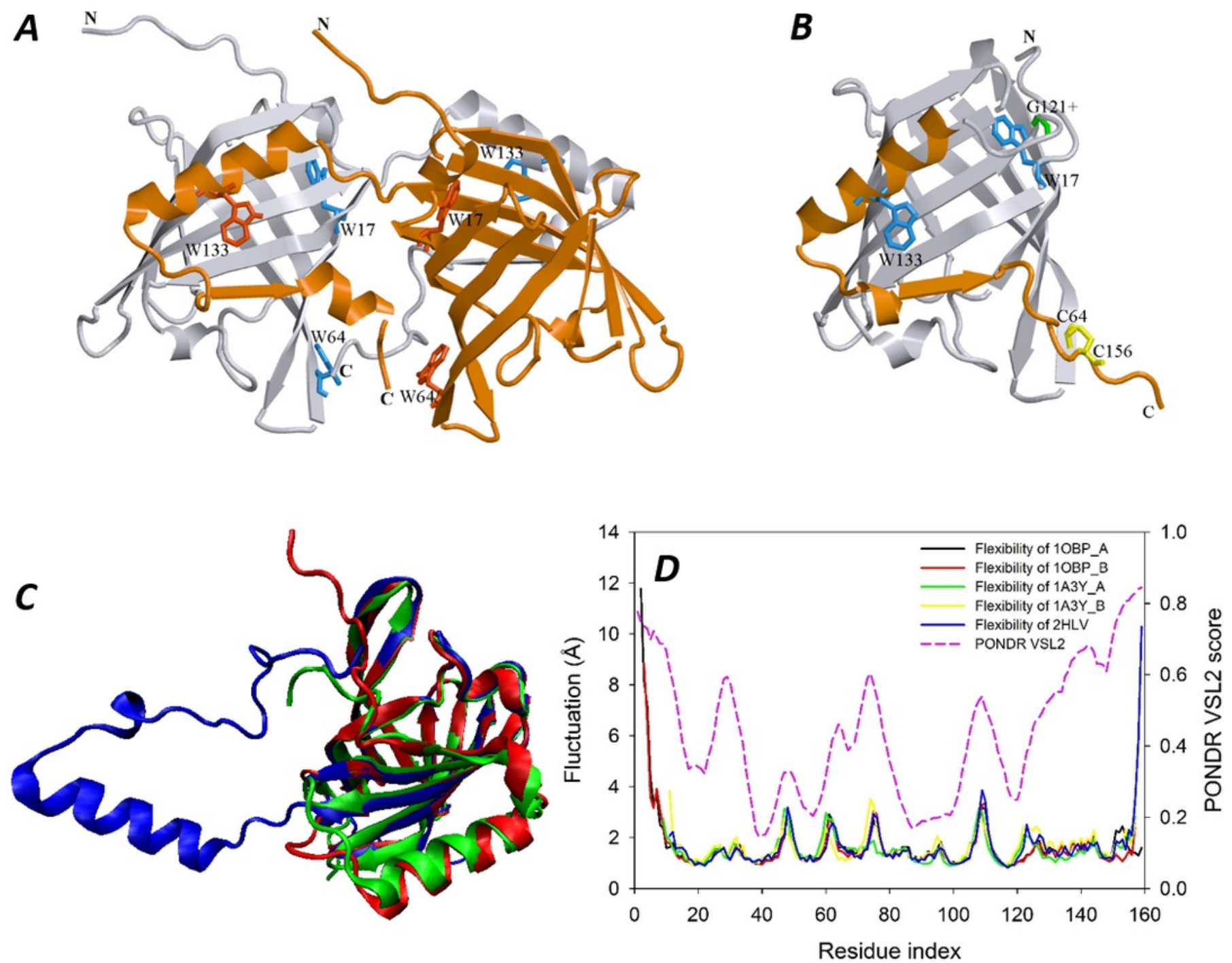
2

The changes of hydrodynamic dimensions of recombinant bOBP ( $A$ and $B$ ) and its complex with ligand bOBP/OCT ( $C$ and $D$ ) in different structural states.

The elution profiles for $\mathrm{BOBP}$ and $\mathrm{BOBP} / \mathrm{OCT}$ were recorded during the protein denaturation $(\boldsymbol{A}$ and $\boldsymbol{C})$ and renaturation from unfolded states $(\boldsymbol{B}$ and $\boldsymbol{D})$ induced by $\mathrm{GdnHCl}$. The elution profiles for bOBP were measured after pre-incubation of the protein and the solution of $\mathrm{GdnHCl}$ in desired concentration for $24 \mathrm{~h}(\boldsymbol{A}$ and $\boldsymbol{B})$, while in the case of bOBP/OCT the incubation time was extended to 72 - $84 \mathrm{~h}$ for denaturation $(\boldsymbol{C})$ and 6 days for renaturation (D). The figures on the curves are the $\mathrm{GdnHCl}$ concentrations.

recombinant bOBP

B

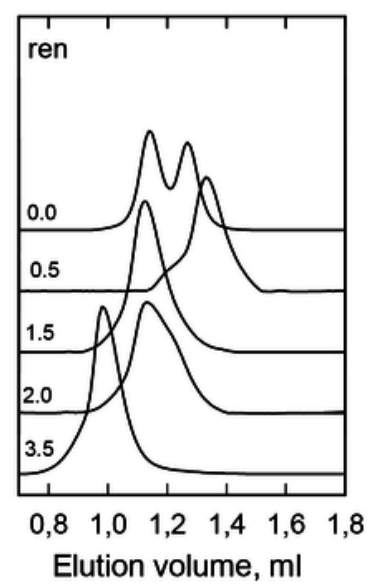

$A$

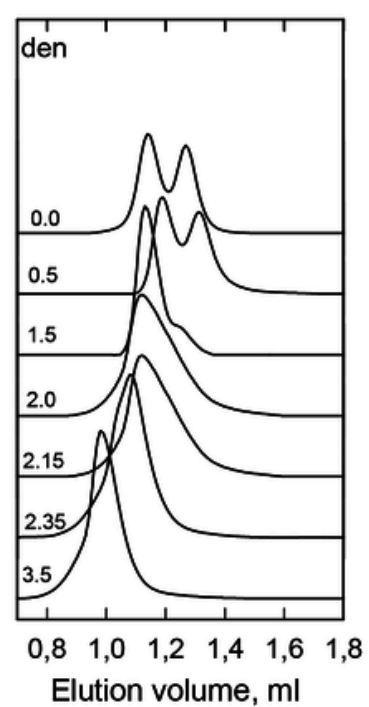

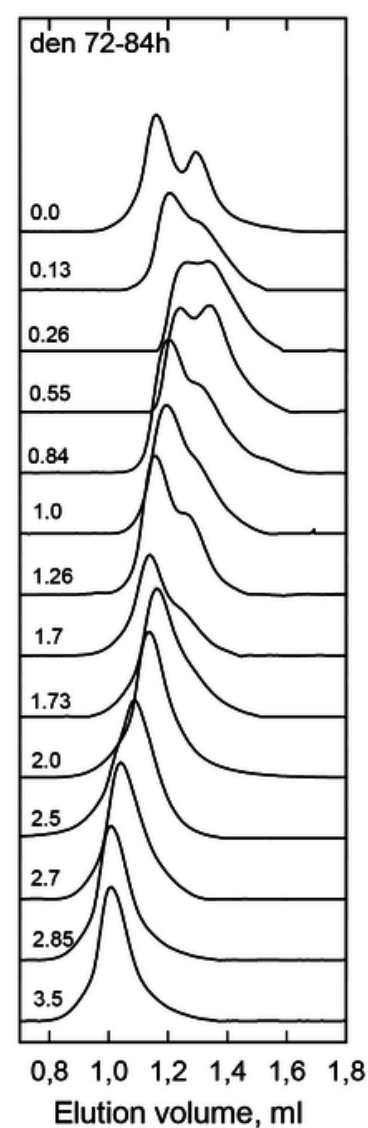

recombinant bOBP/OCT

$D$

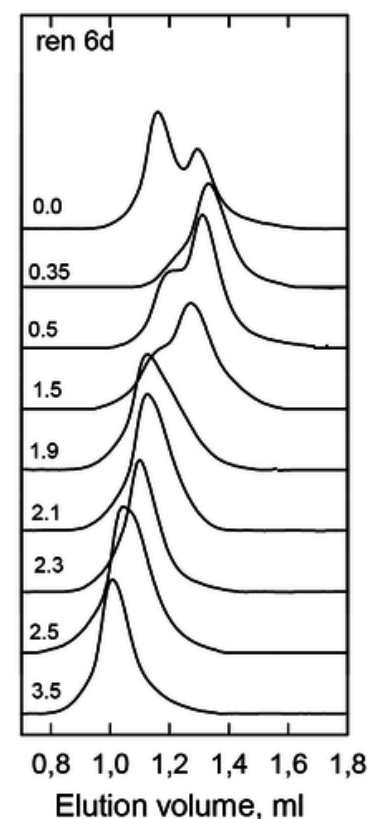




\section{3}

bOBP and bOBP/OCT conformational changes induced by $\mathrm{GdnHCl}$.

$\boldsymbol{A}$ : changes in fluorescence intensity at $320 \mathrm{~nm}, \lambda_{\mathrm{ex}}=297 \mathrm{~nm} ; \boldsymbol{B}$ : changes in parameter $A$, $\lambda_{\mathrm{ex}}=297 \mathrm{~nm} ; \boldsymbol{C}$ : changes in fluorescence anisotropy at the emission wavelength $365 \mathrm{~nm}$, $\mathrm{I}_{\mathrm{ex}}=297 \mathrm{~nm} ; \boldsymbol{D}$ : changes in the ellipticity at $222 \mathrm{~nm} ; \boldsymbol{E}$ : changes in the ANS fluorescence intensity at $\lambda_{\mathrm{ex}}=365 \mathrm{~nm}, \lambda_{\mathrm{em}}=480 \mathrm{~nm}$. The statistical errors for fluorescence measurements were assessed and were shown to fall within the range of $0.2-1 \%$. The measurements were preceded by incubating the protein in a solution with the appropriate $\mathrm{GdnHCl}$ concentration at $4 \stackrel{\circ}{ } \mathrm{C}$ for 24 (red circles) in the case of bOBP. The open symbols indicate unfolding, whereas the closed symbols represent refolding. While studying the folding of bOBP/OCT (squares), the solution of complex of the protein with its ligand were incubated in a solution with the appropriate $\mathrm{GdnHCl}$ concentration at $4 \stackrel{\circ}{\mathrm{C}}$ for less than $24 \mathrm{~h}$ (open brown squares), up to 120 $\mathrm{h}$ (open pink squares) at the protein denaturation, and $1 \mathrm{~h}$ (closed brown squares) and $72 \mathrm{~h}$ 30 days (closed pink squares) at the protein renaturation. 


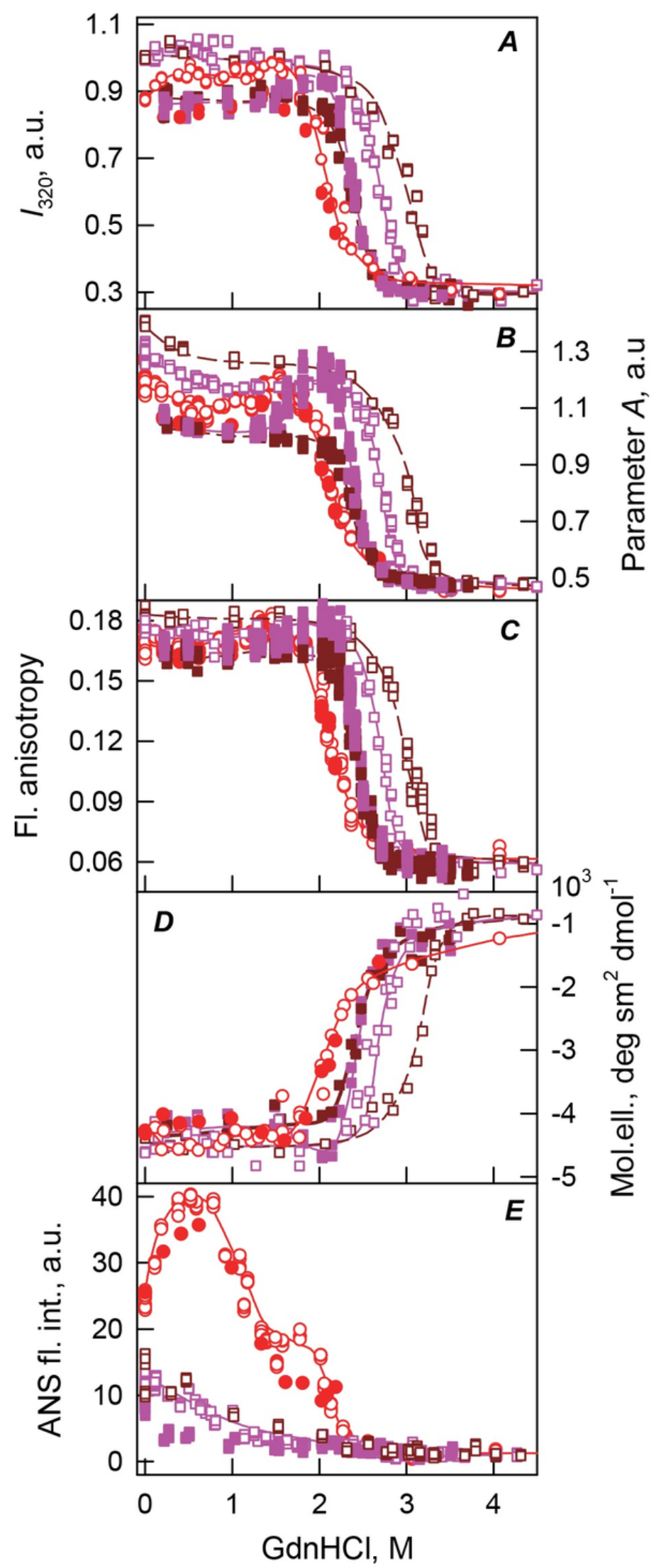




\section{4}

The changes of hydrodynamic dimensions of recombinant bOBP-Gly121+ $(A$ and $B)$ and its complex with ligand bOBP-Gly $121+/ O C T(C$ and $D$ ) in different structural states.

The elution profiles for bOBP-Gly121+ and bOBP-Gly121+/OCT were recorded during the protein denaturation ( $\boldsymbol{A}$ and $\boldsymbol{C}$ ) and renaturation from unfolded states ( $\boldsymbol{B}$ and $\boldsymbol{D}$ ) induced by $\mathrm{GdnHCl}$. The elution profiles for bOBP-Gly121+ were measured after pre-incubation of the protein and the solution of $\mathrm{GdnHCl}$ in desired concentration for $24 \mathrm{~h}(\boldsymbol{A}$ and $\boldsymbol{B})$, while in the case of bOBP-Gly121+/OCT the incubation time was extended to $72-84 \mathrm{~h}$ for denaturation $(\boldsymbol{C})$ and 6 days for renaturation $(\boldsymbol{D})$. The figures on the curves are the $\mathrm{GdnHCl}$ concentrations. 


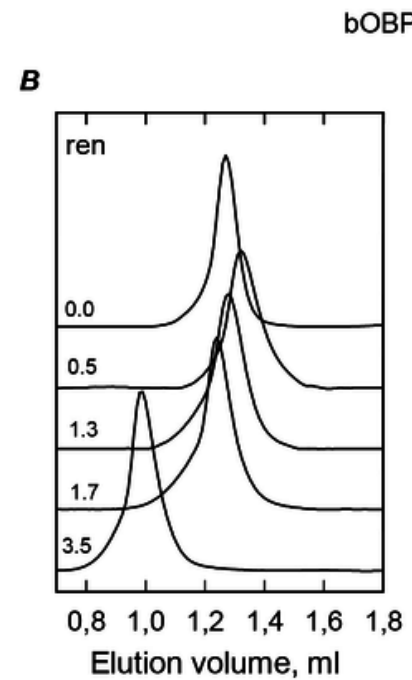

A

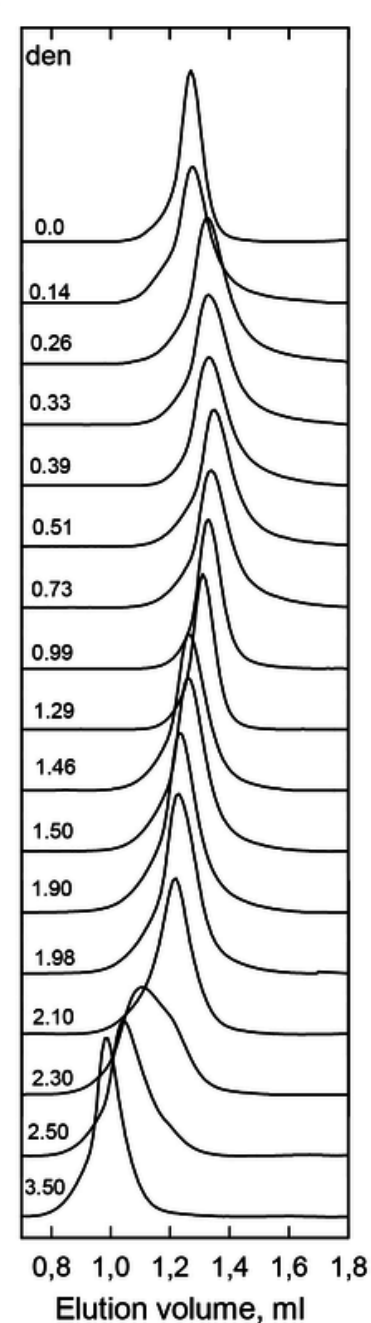

bOBP-Gly121+/OCT

C

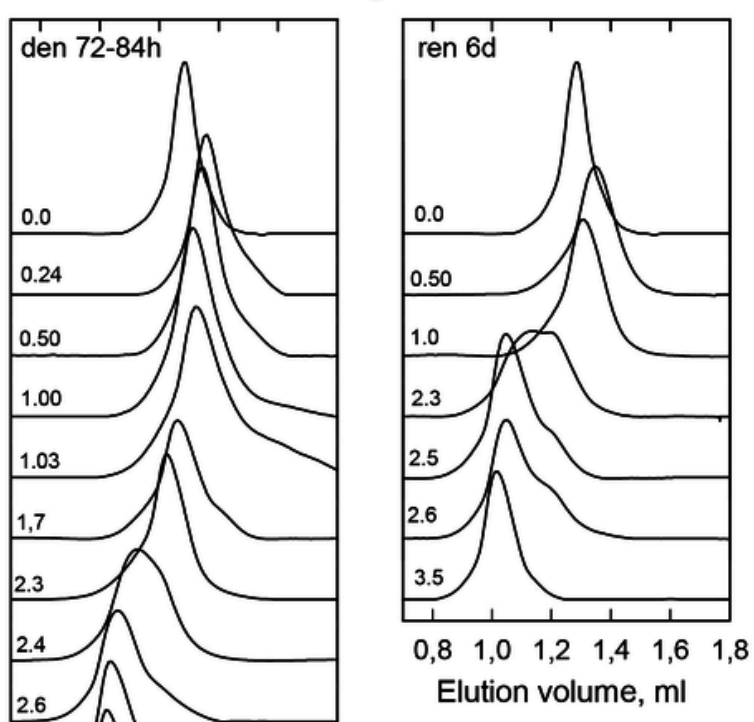




\section{5}

Figure 4. bOBP-Gly $121+$ and bOBP-Gly $121+/ O C T$ conformational changes induced by $\mathrm{GdnHCl}$.

$\boldsymbol{A}$ : changes in fluorescence intensity at $320 \mathrm{~nm}, \lambda_{\mathrm{ex}}=297 \mathrm{~nm}$; $\boldsymbol{B}$ : changes in parameter $A$, $\lambda_{\mathrm{ex}}=297 \mathrm{~nm} ; \boldsymbol{C}$ : changes in fluorescence anisotropy at the emission wavelength $365 \mathrm{~nm}$, $\mathrm{I}_{\mathrm{ex}}=297 \mathrm{~nm} ; \boldsymbol{D}$ : changes in the ellipticity at $222 \mathrm{~nm} ; \boldsymbol{E}$ : changes in the ANS fluorescence intensity at $\lambda_{\mathrm{ex}}=365 \mathrm{~nm}, \lambda_{\mathrm{em}}=480 \mathrm{~nm}$. The measurements were preceded by incubating the protein in a solution with the appropriate $\mathrm{GdnHCl}$ concentration at $4 \stackrel{\circ}{\circ} \mathrm{C}$ for 24 (dark green circles) in the case of bOBP-Gly121+. The open symbols indicate unfolding, whereas the closed symbols represent refolding. While studying the folding of bOBP-Gly $121+/ O C T$ (squares), the solution of complex of the protein with its ligand were incubated in a solution with the appropriate $\mathrm{GdnHCl}$ concentration at $4 \stackrel{\circ}{\mathrm{C}}$ for less than $24 \mathrm{~h}$ (open dark yellow squares), up to $72 \mathrm{~h}$ (open light green squares) at the protein denaturation, and $1 \mathrm{~h}$ (closed dark yellow squares) and $72 \mathrm{~h}$ (closed light green squares) at the protein renaturation. 


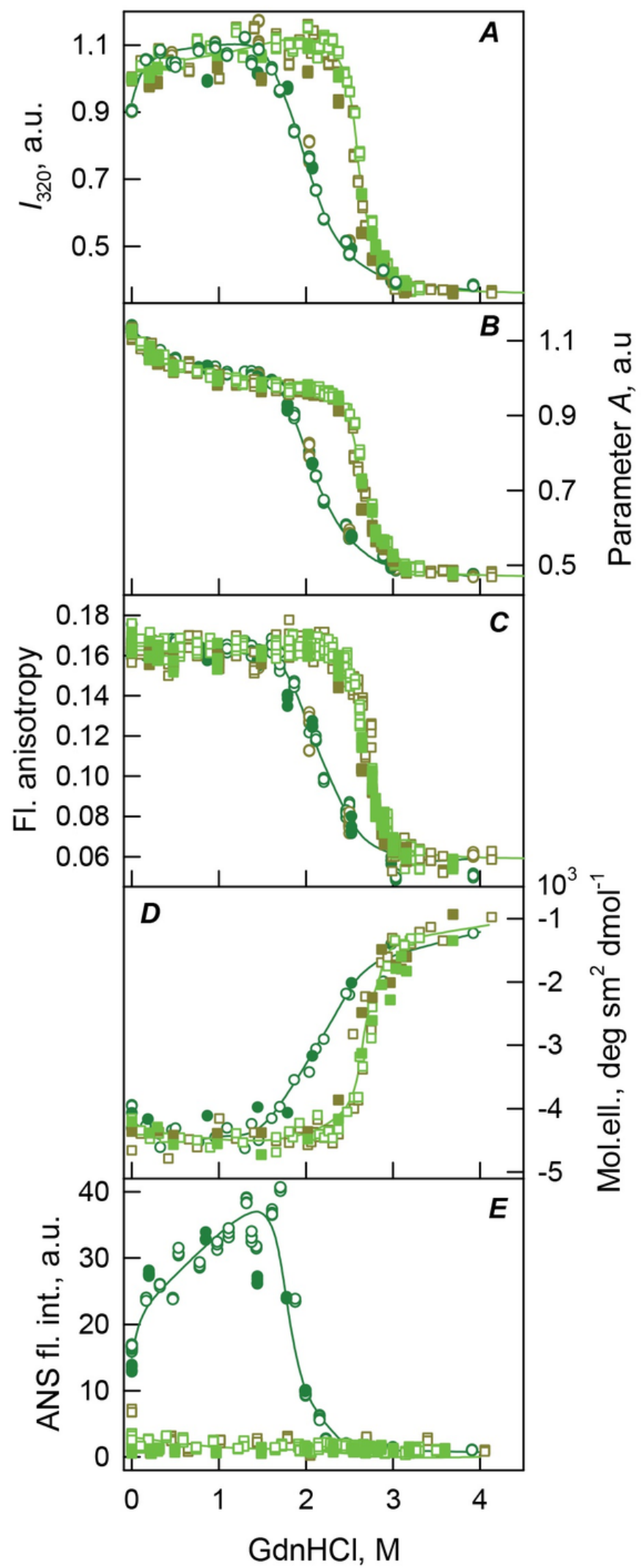


6

The changes of hydrodynamic dimensions of recombinant GCC-bOBP ( $A$ and $B$ ) and its complex with ligand GCC-bOBP/OCT ( $C$ and $D$ ) in different structural states.

The elution profiles for GCC-bOBP and GCC-bOBP/OCT were recorded during the protein denaturation ( $\boldsymbol{A}$ and $\boldsymbol{C}$ ) and renaturation from unfolded states ( $\boldsymbol{B}$ and $\boldsymbol{D}$ ) induced by $\mathrm{GdnHCl}$. The elution profiles for GCC-bOBP were measured after pre-incubation of the protein and the solution of $\mathrm{GdnHCl}$ in desired concentration for $24 \mathrm{~h}(\boldsymbol{A}$ and $\boldsymbol{B})$, while in the case of GCCbOBP/OCT the incubation time was extended to $72-84 \mathrm{~h}$ for denaturation $(\boldsymbol{C})$ and 6 days for renaturation $(\boldsymbol{D})$. The figures on the curves are the $\mathrm{GdnHCl}$ concentrations. 

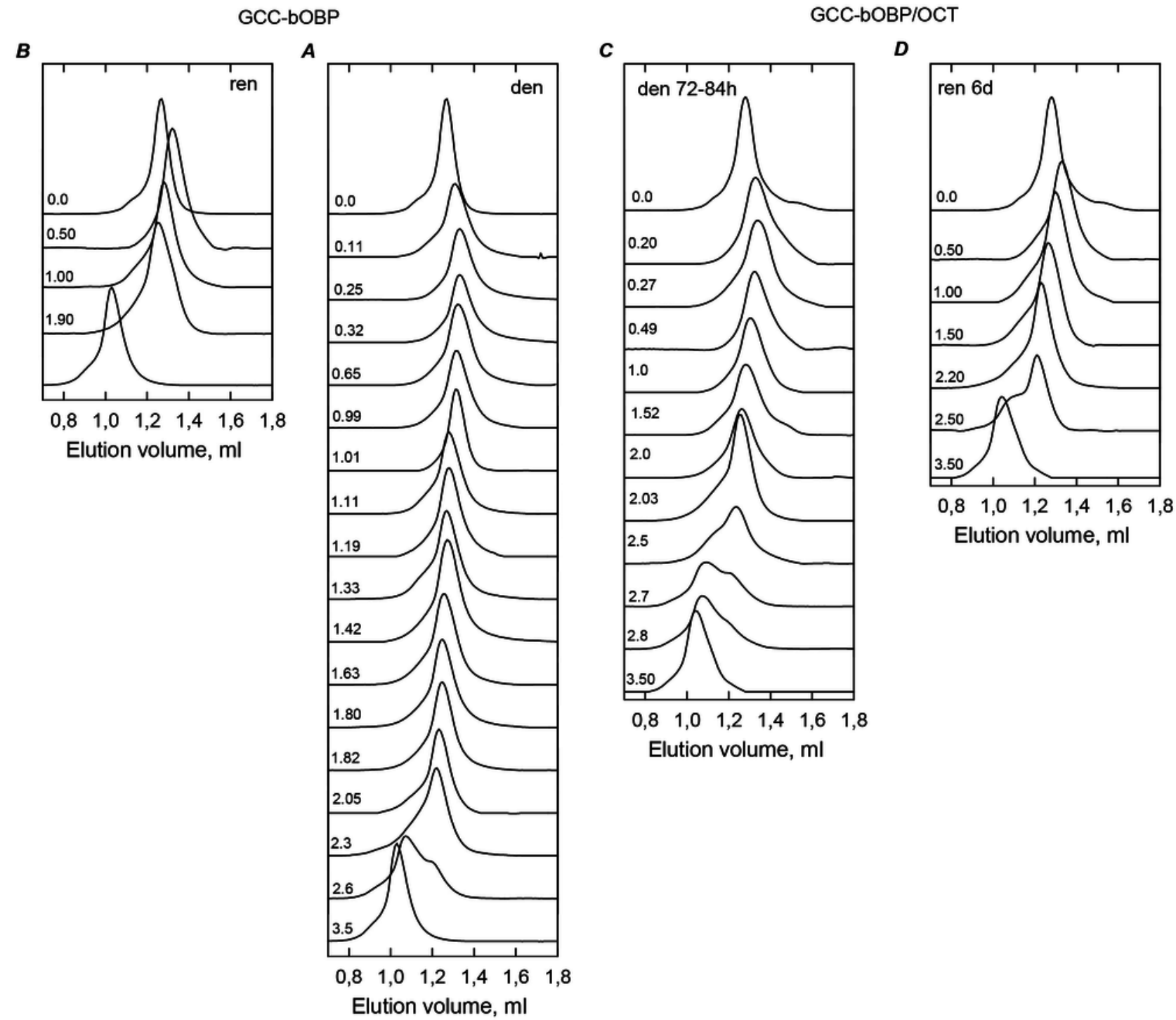


\section{7}

GCC-bOBP and GCC-bOBP/OCT conformational changes induced by GdnHCl.

$\boldsymbol{A}$ : changes in fluorescence intensity at $320 \mathrm{~nm}, \lambda_{\mathrm{ex}}=297 \mathrm{~nm} ; \boldsymbol{B}$ : changes in parameter $A$, $\lambda_{\mathrm{ex}}=297 \mathrm{~nm}$; $\boldsymbol{C}$ : changes in fluorescence anisotropy at the emission wavelength $365 \mathrm{~nm}$, $\mathrm{l}_{\mathrm{ex}}=297 \mathrm{~nm} ; \boldsymbol{D}$ : changes in the ellipticity at $222 \mathrm{~nm} ; \boldsymbol{E}$ : changes in the ANS fluorescence intensity at $\lambda_{\mathrm{ex}}=365 \mathrm{~nm}, \lambda_{\mathrm{em}}=480 \mathrm{~nm}$. The measurements were preceded by incubating the protein in a solution with the appropriate $\mathrm{GdnHCl}$ concentration at $4 \stackrel{\circ}{\mathrm{C}}$ for 24 (blue circles) in the case of GCC-bOBP. The open symbols indicate unfolding, whereas the closed symbols represent refolding. While studying the folding of GCC-bOBP/OCT (squares), the solution of complex of the protein with its ligand were incubated in a solution with the appropriate $\mathrm{GdnHCl}$ concentration at $4 \stackrel{\circ}{\circ} \mathrm{C}$ for less than $24 \mathrm{~h}$ (open dark blue squares), up to $72 \mathrm{~h}$ (open light blue squares) at the protein denaturation, and $1 \mathrm{~h}$ (closed dark blue squares) and $72 \mathrm{~h}$ (closed light blue squares) at the protein renaturation. 


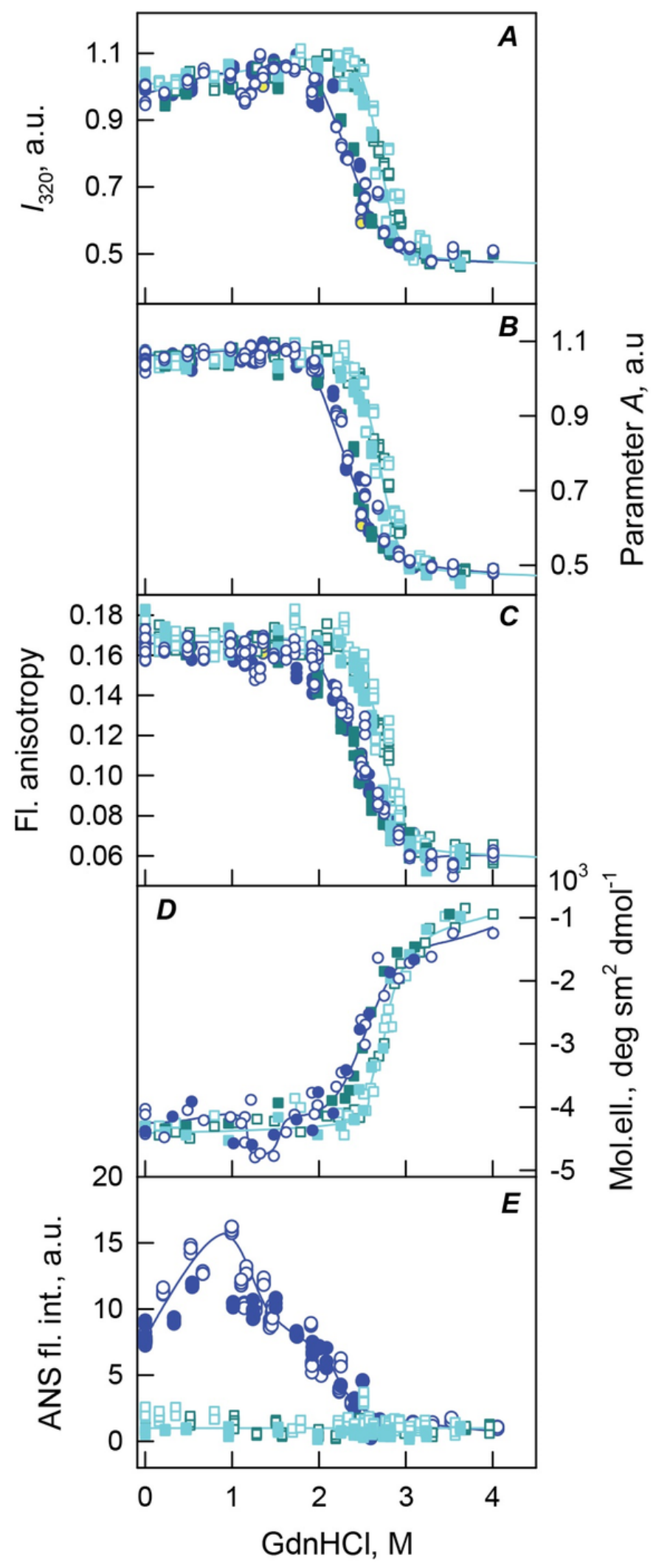

